\title{
Prevalence of pre hypertension and its association to risk factors for cardiovascular diseases among male undergraduate students in Chennai
}

\author{
M. Logaraj*, R. S. Dinesh Madhavan, R. Balaji
}

Department of Community Medicine, SRM Medical College Hospital \& Research Centre, Kattankulathur-603203, India

Received: 25 December 2015

Accepted: 23 January 2016

*Correspondence:

Dr. M. Logaraj,

E-mail: mlogaraj@gmail.com

Copyright: ( $)$ the author(s), publisher and licensee Medip Academy. This is an open-access article distributed under the terms of the Creative Commons Attribution Non-Commercial License, which permits unrestricted non-commercial use, distribution, and reproduction in any medium, provided the original work is properly cited.

\section{ABSTRACT}

Background: Recent studies have documented an increase in the risk of cardiovascular diseases (CVD) and a high rate of progression to hypertension in persons with pre hypertension.

Methods: A cross-sectional study was conducted among students of a university situated in the suburban area of Chennai with an objective to estimate the prevalence of pre-hypertension among male students and to assess the association between pre hypertension and risk factors for cardiovascular diseases. Among 403 students studied which included 200 medical and 203 engineering students with the help of pre tested structured questionnaire and measurements of height, weight, blood pressure; and postprandial blood glucose were carried out as per standard procedure.

Results: About $30.3 \%$ of the students consume alcohol. The prevalence of pre hypertension and hypertension was $49.6 \%$ and $19.1 \%$ among male students. The prevalence of pre-hypertension was higher in medical students $(51.5 \%)$ compared to engineering students $(47.8 \%)$. Higher risk of being pre hypertensive were noted above the age of 20 years $(\mathrm{OR}=4.32)$, fruit intake less than 3 days a week $(\mathrm{OR}=1.03)$, smokers $(\mathrm{OR}=1.13)$, alcohol intake $(\mathrm{OR}=1.56)$, lack of physical exercise $(\mathrm{OR}=1.90)$, BMI of more than $25 \mathrm{~kg} / \mathrm{m}^{2}(\mathrm{OR}=1.99)$. But statistically significant difference was noted between pre hypertensive and normotensive for age $(\mathrm{p}<0.0001)$, lack of physical exercise $(\mathrm{p}=0.004)$ and BMI ( $\mathrm{p}=0.015)$.

Conclusions: In conclusion higher prevalence of smoking, alcohol intake, lack of physical exercise, overweight and increased waist circumference and postprandial blood sugar more than $140 \mathrm{mg} / \mathrm{dl}$ was noted among pre-hypertensive compared to normotensive.

Keywords: Cardiovascular diseases, Pre hypertension, Risk factors, Undergraduate students

\section{INTRODUCTION}

Recent studies have documented an increase in the risk of cardiovascular diseases (CVD) and a high rate of progression to hypertension in persons with systolic blood pressures (SPB) between 120 to $139 \mathrm{~mm} \mathrm{Hg}$ and/or diastolic blood pressures (DBP) between 80 and $89 \mathrm{~mm}$ $\mathrm{Hg}^{1,2}$ Liszka HA et al reported pre-hypertension is associated with increased risk of major cardiovascular events, independent of other cardiovascular risk factors. ${ }^{3}$ Laura $\mathrm{P}$ et al reported nearly one fifth of the pre hypertensive will progress to clinical hypertension over a period of 4 years with higher incidence of progression to hypertension in individuals with higher levels of prehypertension. ${ }^{4}$ Hypertension appears to cluster with other risk factors like obesity, glucose intolerance, dyslipaedimia etc. ${ }^{5,6}$ Pre-hypertension also has been associated with higher cardiovascular risk. In addition, the longitudinal data obtained from the Framingham heart study have indicated that pre hypertensive were associated with more than twofold increase in relative risk from CVD as compared with those with normal 
BP. ${ }^{7,8}$ Much study has not been done on cardiovascular risk factors associated with blood pressure (BP) among college young adults in Indian population. However, there were only few studies done on adult population on the prevalence and risk factors for pre hypertension in Indians adults above the age of 20 years. ${ }^{9,10}$ Demonstration of such risk factors of CVD among pre hypertensive is important for formulating risk reduction strategies particularly in individuals with stage I hypertension or pre hypertension which are widely prevalent than severe stages of hypertension. The objective of our study was to estimate the prevalence of pre-hypertension among undergraduate male students and to assess the association between pre hypertension and some known risk factors for CVD, specifically pre diabetes, overweight or obesity, waist circumference, physical activity, smoking, vegetables and fruit intake.

\section{METHODS}

A cross-sectional study was conducted among male medical and engineering undergraduate students of a university situated in the suburban area of Chennai. A total of 403 students was studied which included 200 medical and 203 engineering students. Recent studies done on the prevalence of pre hypertension in India and other countries showed a prevalence of $46.1 \%$ to $58.7 \%$ among undergraduate students. ${ }^{11-13}$ Based on these studies the prevalence of pre hypertension was taken as $50 \%$ to calculate sample size at 5\% significant level and $10 \%$ allowable error. The sample size was worked out using the formula $\mathrm{n}=4 \mathrm{pq} / \mathrm{L}^{2}$ which came to 400 . Undergraduate students both medical and engineering from first year of academic study to 4th year were invited to take part. There were 22, 109, 43 and 29 students from engineering and 50, 84, 14 and 52 medical streams who volunteered and gave informed consent.

The information on selected socio-demographic variables, tobacco and alcohol use, dietary intake, physical activity and treatment history for diabetes and hypertension were collected with the help of pre tested structured questionnaire. The physical examination of all the participants included measurements of height, weight, blood pressure; resting pulse rate and postprandial blood glucose was carried out. The height was measured using a portable stadiometer. The participants were asked to stand in erect posture without foot wear with their feet together and to look straight. The stadiometer's measuring tip was lowered to the head and height was measured.

The weights of the participants were measured by using a portable electronic weighing machine. The participants were asked to stand still on the platform of the weighing machine. The cloth they were wearing was allowed to be worn but without the foot wear. The body mass index was measured using the formula weight $(\mathrm{kg}) / \mathrm{height}(\mathrm{m})^{2}$. overweight was defined as a BMI greater than or equal to $25 \mathrm{~kg} / \mathrm{m}^{2}$, while obesity is defined as BMI greater than or equal $30 \mathrm{~kg} / \mathrm{m}^{2}{ }^{14}$ Increased (high-risk) waist circumference is defined as recommended by Lean et al, ${ }^{15}$ as greater than $94 \mathrm{~cm}$ in men. Increased waist to hip ratio was defined as greater than or equal to 0.95 for males.

Postprandial blood sugar was measured using glucometer (accutrend plus). It was ensured before taking capillary blood sample that the students had not taken any food two hour before testing. A person with a postprandial blood glucose concentration between 140-199 mg/dl was considered as pre diabetic, over $11.0 \mathrm{mmol} / \mathrm{l}(200 \mathrm{mg} / \mathrm{dl})$ or if he or she was taking insulin or oral hypoglycemic drugs said to have diabetes.

Blood pressure was recorded in the sitting position in the left arm to the nearest $1 \mathrm{mmHg}$ using an electronic Omron machine (Omron Corporation, Tokyo, Japan). Two readings were taken, one before start of interview and other at the end of the interview and the mean of the 2 readings was used for analysis. Pre hypertension was defined according to JNC 7 criteria as having either a systolic blood pressure of 120 to $139 \mathrm{mmhg}$ and/or diastolic blood pressure of 80 to $89 \mathrm{~mm} \mathrm{Hg}$ in persons who were not on treatment for hypertension. Hypertension was defined according to JNC 7 criteria as having an untreated systolic blood pressure (BP) of greater than or equal to $140 \mathrm{~mm} \mathrm{Hg}$ or diastolic BP greater than or equal to $90 \mathrm{~mm} \mathrm{Hg}$ or being on medication for hypertension. Normal blood pressure was defined as having both a systolic BP of $<120 \mathrm{~mm} \mathrm{Hg}$ and a diastolic $\mathrm{BP}$ of $<80 \mathrm{~mm} \mathrm{Hg}$ in the absence of antihypertensive medication ${ }^{9}$ or was already on treatment for hypertension. All current smokers and those who quit smoking less than 1 year before the assessment were considered smokers for assessing cardiovascular risk. The same way all current alcoholics and those who quit alcohol less than 1 year before the assessment were considered alcoholics.

Study was carried out after obtaining institutional ethics committee approval. Data was analyzed using the standard statistical software packages. Descriptive data were presented as percentages and unadjusted odds ratios (OR) to measure the strength of association and $95 \%$ confidence intervals (CI) were calculated. Chi-square test was used to lend statistical support to prove associations between categorical variables.

\section{RESULTS}

This cross sectional study was conducted among 403 students consisting of 203 engineering students and 200 medical students in a university in a suburban area of Chennai. The mean age of the participants was $19.56 \pm 1.67$ years. The mean height and weight of the aprtic0ants were $176.55 \pm 80.94 \mathrm{~cm}$ and $70.71 \pm 13.49 \mathrm{~kg}$ respectively. The mean systolic and diastolic blood pressure was $125.80 \pm 10.03 \mathrm{~mm}$ of $\mathrm{Hg}$ and $78.96 \pm 11.75$ $\mathrm{mm}$ of $\mathrm{Hg}$. The mean blood sugar level was $95.62 \pm 16.09$ $\mathrm{mg} / \mathrm{dl}$. The average intake of fruits and vegetable per 
week were $4.34 \pm 3.47$ days and $6.55 \pm 4.39$ days respectively.

Table 1 depicts the prevalence of CVD risk factors among male undergraduate students. $26.1 \%$ were above the age of 20 years. Fruit and vegetable intake for less than 3 days a week was $47.6 \%$ and $24.3 \%$ respectively. Vegetarians were $15.4 \%$. Use of smoke and smokeless tobacco were $27.3 \%$ and $3 \%$ respectively. About $30.3 \%$ of the university students consume alcohol. Nearly $54.1 \%$ of the students take regular exercise. In the anthropometric measurement $29 \%$ were overweight and5.7\% were obese, $24.8 \%$ were with waist circumference above 90 centimeters. About $4.2 \%$ had postprandial blood sugar more than $140 \mathrm{mg} / \mathrm{dl}$. The prevalence of pre-hypertension and hypertension was $49.6 \%$ and $19.1 \%$ among male undergraduate students. Higher number of male engineering students were consuming fruits less than 3 days a week, smokers, consume alcohol, postprandial blood sugar of more than $140 \mathrm{mg} / \mathrm{dl}$ and hypertensive. Statistically significant differences were found for smokers $(\mathrm{p}<0.001)$, alcohol intake $(p<0.001)$ and postprandial blood sugar of more than $140 \mathrm{mg} / \mathrm{dl}(\mathrm{p}=0.02)$. Higher number of male medical students consumes vegetables for less than 3 days a week; take mixed diet, overweight, waist circumference more than $90 \mathrm{~cm}$ and pre hypertensive. Statistically significant difference were found for waist circumference more than $90 \mathrm{~cm}(\mathrm{p}<0.001)$.

Table 2 depicts, overall prevalence of normotensive, prehypertension and hypertension among male students was 126/403 (31.3\%), 200/403 (49.6\%) and 77/403 (19.1\%) respectively. The prevalence of pre-hypertension was higher in medical students $(51.5 \%)$ compared to engineering students $(47.8 \%)$ and it was not statistically significant. The prevalence of pre hypertension was higher in above the age of 20 years $(29.5 \%)$, vegetable intake less than 3 days a week $(26.5 \%)$, smokers $(29.5 \%)$, alcoholics $(31 \%)$ those did not practice regular exercise $(47 \%)$ and postprandial blood sugar more than $140 \mathrm{mg} / \mathrm{dl}$ (3\%).those did not practice regular exercise $(47 \%)$ and postprandial blood sugar more than $140 \mathrm{mg} / \mathrm{dl}(3 \%)$. Significant association was found for alcohol intake $(\mathrm{p}=0.03)$, increased BMI $(\mathrm{p}<0.001)$ and increased waist circumference $(\mathrm{p}=0.003)$.

Table 1: Distribution of risk factors of CVD among male engineering and medical students.

\begin{tabular}{|c|c|c|c|c|c|}
\hline Risk Factor & $\begin{array}{l}\text { Engineering } \\
(\mathrm{N}=\mathbf{2 0 3})\end{array}$ & $\begin{array}{l}\text { Medical } \\
(\mathrm{N}=\mathbf{2 0 0})\end{array}$ & $\begin{array}{l}\text { Total } \\
(\mathrm{N}=403)\end{array}$ & $x^{2}$ & $\begin{array}{l}\mathbf{P} \\
\text { Value }\end{array}$ \\
\hline Age $<20$ Years & $165(81.3)$ & $133(66.5)$ & $298(73.9)$ & \multirow[b]{2}{*}{11.4} & \multirow[b]{2}{*}{0.001} \\
\hline Age $>20$ years & $38(18.7)$ & $67(33.5)$ & $105(26.1)$ & & \\
\hline Fruit intake $<3$ days/week & $106(52.2)$ & $86(43.0)$ & $192(47.6)$ & \multirow[b]{2}{*}{3.43} & \multirow[b]{2}{*}{0.064} \\
\hline Fruit intake/ >3 Days/week & $97(47.8)$ & $114(57.0)$ & $211(52.4)$ & & \\
\hline Vegetable intake $<3$ days/week & $45(22.2)$ & $53(26.5)$ & $98(24.3)$ & \multirow[b]{2}{*}{1.03} & \multirow[b]{2}{*}{0.311} \\
\hline Vegetable intake $>3$ days/week & $158(77.8)$ & $147(73.5)$ & $305(75.7)$ & & \\
\hline Diet-vegetarian & $40(19.7)$ & $22(11.0)$ & $62(15.4)$ & \multirow[b]{2}{*}{5.86} & \multirow[b]{2}{*}{0.015} \\
\hline Diet - mixed & $163(80.3)$ & $178(89.0)$ & $341(84.6)$ & & \\
\hline Smokers & $74(36.5)$ & $36(18.0)$ & $110(27.3)$ & \multirow[b]{2}{*}{17.3} & \multirow[b]{2}{*}{$<0.001$} \\
\hline Non smokers & $129(63.5)$ & $164(82.0)$ & $293(72.7)$ & & \\
\hline Use smokeless tobacco & $8(3.9)$ & $4(2.0)$ & $12(3.0)$ & \multirow[b]{2}{*}{1.31} & \multirow[b]{2}{*}{0.252} \\
\hline Did not use smokeless tobacco & $195(96.1)$ & $196(98.0)$ & $391(97.0)$ & & \\
\hline $\mathrm{H} / \mathrm{O}$ alcohol intake & $78(38.4)$ & $44(22.0)$ & $122(30.3)$ & \multirow[b]{2}{*}{12.9} & \multirow[b]{2}{*}{0.000} \\
\hline No $\mathrm{H} / \mathrm{O}$ of alcohol intake & $125(61.6)$ & $156(78.0)$ & $281(69.7)$ & & \\
\hline $\mathrm{H} / \mathrm{O}$ regular exercise & $110(54.2)$ & $108(54.0)$ & $218(54.1)$ & \multirow[b]{2}{*}{0.142} & \multirow[b]{2}{*}{0.970} \\
\hline No H/O regular exercise & $93(45.8)$ & $92(46.0)$ & $185(45.9)$ & & \\
\hline $\mathrm{BMI}<25 \mathrm{~kg} / \mathrm{m}^{2}$ & $140(68.9)$ & $123(61.5)$ & $263(65.3)$ & \multirow[b]{3}{*}{3.63} & \multirow[b]{3}{*}{0.163} \\
\hline BMI- $25-29.99 \mathrm{~kg} / \mathrm{m}^{2}$ & $55(27.2)$ & $62(31.0)$ & $117(29.0)$ & & \\
\hline $\mathrm{BMI}>30 \mathrm{~kg} / \mathrm{m}^{2}$ & $8(3.9)$ & $15(7.5)$ & $23(5.7)$ & & \\
\hline Waist circumference $<90 \mathrm{~cm}$ & $173(85.2)$ & $130(65.0)$ & $303(75.2)$ & \multirow[b]{2}{*}{22.1} & \multirow[b]{2}{*}{0.000} \\
\hline Waist circumference $>90 \mathrm{~cm}$ & $30(14.8)$ & $70(35.0)$ & $100(24.8)$ & & \\
\hline Blood sugar $<140 / \mathrm{m} / \mathrm{dl}$ & $190(93.6)$ & $196(98.0)$ & $386(95.8)$ & \multirow[b]{2}{*}{4.84} & \multirow{2}{*}{0.027} \\
\hline Blood sugar $>140 \mathrm{mg} / \mathrm{dl}$ & $13(6.4)$ & $4(2.0)$ & $17(4.2)$ & & \\
\hline Normal BP & $59(29.1)$ & $67(33.5)$ & $126(31.3)$ & \multirow{3}{*}{4.42} & \multirow{3}{*}{0.11} \\
\hline Pre Hypertension & $97(47.8)$ & $103(51.5)$ & $200(49.6)$ & & \\
\hline Hypertension & $47(23.1)$ & $30(15.0)$ & $77(19.1)$ & & \\
\hline
\end{tabular}


Table 2: Prevalence of risk factors of pre-hypertension among undergraduate students.

\begin{tabular}{|c|c|c|c|c|c|}
\hline Risk Factors & $\begin{array}{c}\text { Normal BP } \\
(\mathrm{N}=126)\end{array}$ & $\begin{array}{l}\text { Pre HTN } \\
(\mathrm{N}=\mathbf{2 0 0})\end{array}$ & $\begin{array}{l}\text { HTN } \\
(\mathrm{N}=77)\end{array}$ & $\chi^{2}$ & P value \\
\hline Age $>20$ Years & $24(19.1)$ & $59(29.5)$ & $22(28.6)$ & 4.70 & 0.09 \\
\hline Engineering students & $59(46.8)$ & $97(48.5)$ & $47(61.0)$ & \multirow{2}{*}{4.42} & \multirow{2}{*}{0.110} \\
\hline Medical students & $67(53.2)$ & $103(51.5)$ & $30(39.0)$ & & \\
\hline Diet- Mixed & $108(85.7)$ & $167(83.5)$ & $66(85.7)$ & 0.379 & 0.827 \\
\hline Fruit intake $<3$ days/ week & $60(47.6)$ & $89(44.5)$ & $42(54.5)$ & 2.25 & 0.324 \\
\hline Vegetable intake $<3$ days/ week & $29(23.0)$ & $53(26.5)$ & $16(20.8)$ & 1.16 & 0.561 \\
\hline Smokers & $26(20.6)$ & $59(29.5)$ & $25(32.5)$ & 4.34 & 0.114 \\
\hline Use smokeless tobacco & $4(3.2)$ & $3(1.5)$ & $5(6.5)$ & 4.82 & 0.090 \\
\hline $\mathrm{H} / \mathrm{O}$ alcohol intake & $29(23.0)$ & $62(31.0)$ & $31(40.3)$ & 6.83 & 0.033 \\
\hline No H/O regular exercise & $53(42.1)$ & $94(47.0)$ & $38(49.4)$ & 1.21 & 0.545 \\
\hline $\mathrm{BMI}>25 \mathrm{~kg} / \mathrm{m}^{2}$ & $29(23.1)$ & $72(36.0)$ & $39(51.6)$ & 16.4 & 0.000 \\
\hline Waist circumference $>90 \mathrm{~cm}$ & $18(14.3)$ & $56(28.0)$ & $26(33.8)$ & 11.9 & 0.003 \\
\hline Blood sugar $>140 \mathrm{mg} / \mathrm{dl}$ & $1(0.8)$ & $6(3.0)$ & $3(3.9)$ & 2.34 & 0.310 \\
\hline
\end{tabular}

Table 3: Prevalence of risk factors for cardiovascular diseases in pre -hypertensive and hypertensive's among engineering and medical students.

\begin{tabular}{|c|c|c|c|c|c|c|c|c|c|c|}
\hline \multirow[t]{2}{*}{$\begin{array}{l}\text { Risk } \\
\text { Factors }\end{array}$} & \multicolumn{5}{|c|}{ Engineering students ( $\mathrm{N}=\mathbf{2 0 3}$ ) } & \multicolumn{5}{|c|}{ Medical students ( $\mathbf{N}=\mathbf{2 0 0})$} \\
\hline & $\begin{array}{l}\text { Normal BP } \\
(\%) \\
95 \% \text { CI }\end{array}$ & $\begin{array}{l}\text { Pre-HTN } \\
\% \\
95 \% \mathrm{CI}\end{array}$ & $\begin{array}{l}\text { HTN } \\
\% \\
95 \% \mathrm{CI}\end{array}$ & $\chi^{2}$ & $\begin{array}{l}\mathrm{P} \\
\text { value }\end{array}$ & $\begin{array}{l}\text { Normal } \\
\text { BP } \\
\% \\
95 \% \mathrm{CI}\end{array}$ & $\begin{array}{l}\text { Pre-HTN \% } \\
95 \% \text { CI }\end{array}$ & $\begin{array}{l}\text { HTN \% } \\
95 \% \text { CI }\end{array}$ & $\chi^{2}$ & $\begin{array}{l}\mathrm{P} \\
\text { value }\end{array}$ \\
\hline $\begin{array}{l}\text { Age }>20 \\
\text { yrs. }\end{array}$ & $\begin{array}{l}12.1 \\
7.61-16.59\end{array}$ & $\begin{array}{l}22.8 \\
17.03-28.57\end{array}$ & $\begin{array}{l}20.0 \\
14.5-25.5\end{array}$ & 2.96 & 0.228 & $\begin{array}{l}25.4 \\
19.37-31.43\end{array}$ & $\begin{array}{l}35.9 \\
29.25-42.55\end{array}$ & $\begin{array}{l}43.3 \\
36.43-50.17\end{array}$ & 3.56 & 0.169 \\
\hline $\begin{array}{l}\text { Diet- } \\
\text { Mixed }\end{array}$ & $\begin{array}{l}83.3 \\
78.17-88.43\end{array}$ & $\begin{array}{l}76.1 \\
70.23-81.97\end{array}$ & $\begin{array}{l}84.4 \\
79.41-89.39\end{array}$ & 1.90 & 0.386 & $\begin{array}{l}85.1 \\
80.16-90.04\end{array}$ & $\begin{array}{l}91.3 \\
87.39-95.21\end{array}$ & $\begin{array}{l}90.0 \\
85.84-94.16\end{array}$ & 1.62 & 0.444 \\
\hline $\begin{array}{l}\text { Fruit } \\
\text { intake <3 } \\
\text { days/ } \\
\text { week }\end{array}$ & $\begin{array}{l}51.5 \\
44.62-58.38\end{array}$ & $\begin{array}{l}53.2 \\
46.34-60.06\end{array}$ & $\begin{array}{l}55.5 \\
48.66-62.34\end{array}$ & 0.176 & 0.916 & $\begin{array}{l}46.3 \\
39.39-53.21\end{array}$ & $\begin{array}{l}46.6 \\
39.69-53.51\end{array}$ & $\begin{array}{l}56.7 \\
49.83-63.57\end{array}$ & 1.06 & 0.587 \\
\hline $\begin{array}{l}\text { Vegetable } \\
\text { intake <3 } \\
\text { days/ } \\
\text { week }\end{array}$ & $\begin{array}{l}18.2 \\
12.89-23.51\end{array}$ & $\begin{array}{l}23.9 \\
18.03-29.77\end{array}$ & $\begin{array}{l}24.4 \\
18.9-30.31\end{array}$ & 0.905 & 0.636 & $\begin{array}{l}29.9 \\
23.56-36.24\end{array}$ & $\begin{array}{l}28.2 \\
21.96-34.44\end{array}$ & $\begin{array}{l}33.3 \\
26.77-39.16\end{array}$ & 3.20 & 0.202 \\
\hline Smokers & $\begin{array}{l}34.8 \\
28.25-41.35\end{array}$ & $\begin{array}{l}26.1 \\
20.06-32.14\end{array}$ & $\begin{array}{l}37.7 \\
31.03-44.37\end{array}$ & 2.41 & 0.299 & $\begin{array}{l}8.9 \\
4.95-12.85\end{array}$ & $\begin{array}{l}22.3 \\
16.53-28.07\end{array}$ & $\begin{array}{l}23.3 \\
17.44-29.16\end{array}$ & 5.60 & 0.061 \\
\hline $\begin{array}{l}\mathrm{H} / 0 \text { of } \\
\text { use of } \\
\text { smokeless } \\
\text { tobacco }\end{array}$ & $\begin{array}{l}1.5 \\
-0.17-3.17\end{array}$ & $\begin{array}{l}3.3 \\
0.84-5.76\end{array}$ & $\begin{array}{l}8.9 \\
4.98-12.82\end{array}$ & 4.05 & 0.132 & $\begin{array}{l}4.5 \\
1.63-7.37\end{array}$ & 0.0 & $\begin{array}{l}3.3 \\
0.82-5.78\end{array}$ & 4.47 & 0.107 \\
\hline $\begin{array}{l}\mathrm{H} / \mathrm{O} \\
\text { alcohol } \\
\text { intake }\end{array}$ & $\begin{array}{l}34.8 \\
28.25-41.35\end{array}$ & $\begin{array}{l}39.1 \\
32.39-45.51\end{array}$ & $\begin{array}{l}40.0 \\
33.26-46.74\end{array}$ & 0.404 & 0.817 & $\begin{array}{l}10.4 \\
6.17-14.63\end{array}$ & $\begin{array}{l}24.3 \\
18.36-30.24\end{array}$ & $\begin{array}{l}40.0 \\
33.21-46.79\end{array}$ & 11.2 & 0.004 \\
\hline $\begin{array}{l}\text { No } \mathrm{H} / \mathrm{O} \\
\text { regular } \\
\text { exercise }\end{array}$ & $\begin{array}{l}37.9 \\
31.23-44.57\end{array}$ & $\begin{array}{l}69.6 \\
63.27-75.93\end{array}$ & $\begin{array}{l}55.6 \\
48.77-62.43\end{array}$ & 15.7 & 0.000 & $\begin{array}{l}52.2 \\
45.28-59.12\end{array}$ & $\begin{array}{l}53.4 \\
46.49-60.31\end{array}$ & $\begin{array}{l}60.0 \\
53.21-66.79\end{array}$ & $\begin{array}{l}0.53 \\
3\end{array}$ & 0.766 \\
\hline $\begin{array}{l}\mathrm{BMI}>25 \\
\mathrm{~kg} / \mathrm{m}^{2}\end{array}$ & $\begin{array}{l}21.2 \\
15.58-26.82\end{array}$ & $\begin{array}{l}32.6 \\
26.15-39.05\end{array}$ & $\begin{array}{l}42.2 \\
39.41-48.99\end{array}$ & 5.71 & 0.057 & $\begin{array}{l}22.4 \\
16.62-28.18\end{array}$ & $\begin{array}{l}40.8 \\
33.99-47.61\end{array}$ & $\begin{array}{l}66.7 \\
60.17-73.23\end{array}$ & 8.96 & 0.011 \\
\hline $\begin{array}{l}\text { Waist } \\
\text { circumfer } \\
\text { ence }>90 \\
\mathrm{~cm}\end{array}$ & $\begin{array}{l}13.6 \\
8.88-18.32\end{array}$ & $\begin{array}{l}10.9 \\
6.61-15.19\end{array}$ & $\begin{array}{l}24.4 \\
18.49-30.31\end{array}$ & 4.52 & 0.104 & $\begin{array}{l}16.4 \\
11.27-21.53\end{array}$ & $\begin{array}{l}42.7 \\
35.84-49.56\end{array}$ & $\begin{array}{l}50.0 \\
43.07-56.93\end{array}$ & 15.8 & 0.000 \\
\hline $\begin{array}{l}\text { Blood } \\
\text { sugar } \\
>140 \\
\mathrm{mg} / \mathrm{dl}\end{array}$ & $\begin{array}{l}12.1 \\
7.61-16.59\end{array}$ & $\begin{array}{l}2.2 \\
0.18-4.22\end{array}$ & $\begin{array}{l}6.6 \\
3.18-10.02\end{array}$ & 6.35 & 0.042 & 0.0 & $\begin{array}{l}3.9 \\
1.22-6.58\end{array}$ & 0.0 & 3.84 & 0.146 \\
\hline
\end{tabular}


Table 4: Association between cardiovascular risk factors and pre hypertensive.

\begin{tabular}{|c|c|c|c|c|c|c|c|}
\hline Risk Factors & Normal & $\begin{array}{l}\text { Pre - } \\
\text { HTN }\end{array}$ & $\mathrm{OR}, 95 \% \mathrm{CI}$ & $\begin{array}{l}\chi^{2} \\
\mathbf{P} \text { value }\end{array}$ & HTN & $\begin{array}{l}\text { OR } \\
95 \% \mathrm{CI}\end{array}$ & $\begin{array}{l}\chi^{2} \mathbf{P} \\
\text { value }\end{array}$ \\
\hline Age $<20$ years & 108 & 137 & 1 & \multirow{2}{*}{$\begin{array}{l}27.15 \\
<0.0001\end{array}$} & 53 & 1 & \multirow{2}{*}{$\begin{array}{l}3.04 \\
0.08\end{array}$} \\
\hline Age $>20$ years & 25 & 58 & $\begin{array}{l}4.32 \\
2.449-7.617\end{array}$ & & 22 & $\begin{array}{l}1.793 \\
0.926-3.471\end{array}$ & \\
\hline Diet-vegetarian & 21 & 31 & 1 & \multirow[b]{2}{*}{01} & 10 & 1 & \multirow{2}{*}{$\begin{array}{l}0.23 \\
0.631\end{array}$} \\
\hline Diet-mixed & 112 & 164 & $\begin{array}{l}0.991 \\
0.542-1.814\end{array}$ & & 65 & $\begin{array}{l}1.218 \\
0.540-2.747\end{array}$ & \\
\hline $\begin{array}{l}\text { Fruit intake }>3 \text { days / } \\
\text { week }\end{array}$ & 68 & 98 & 1 & \multirow{2}{*}{$\begin{array}{l}0.02 \\
0.887\end{array}$} & 33 & 1 & \multirow{2}{*}{$\begin{array}{l}0.98 \\
0.322\end{array}$} \\
\hline $\begin{array}{l}\text { Fruit intake <3 days/ } \\
\text { week }\end{array}$ & 65 & 97 & $\begin{array}{l}1.035 \\
0.666-1.609\end{array}$ & & 42 & $\begin{array}{l}1.331 \\
0.753-2.351\end{array}$ & \\
\hline $\begin{array}{l}\text { Vegetable intake >3 } \\
\text { days/week }\end{array}$ & 101 & 144 & 1 & \multirow{2}{*}{$\begin{array}{l}0.18 \\
0.671\end{array}$} & 60 & 1 & \multirow{2}{*}{$\begin{array}{l}0.45 \\
0.502\end{array}$} \\
\hline $\begin{array}{l}\text { Vegetable intake <3 } \\
\text { days/ week }\end{array}$ & 32 & 51 & $\begin{array}{l}1.117 \\
0.671-1.861\end{array}$ & & 15 & $\begin{array}{l}0.789 \\
0.395-1.575\end{array}$ & \\
\hline Non smokers & 104 & 148 & 1 & \multirow[b]{2}{*}{$\begin{array}{l}0.23 \\
0.631\end{array}$} & 51 & 1 & \multirow{2}{*}{$\begin{array}{l}2.63 \\
0.104\end{array}$} \\
\hline Smokers & 29 & 47 & $\begin{array}{l}1.138 \\
0.672-1.927\end{array}$ & & 24 & $\begin{array}{l}1.687 \\
0.893-3.188\end{array}$ & \\
\hline $\begin{array}{l}\text { No } \mathrm{H} / 0 \text { of use of } \\
\text { smokeless tobacco }\end{array}$ & 129 & 192 & 1 & \multirow{2}{*}{$\begin{array}{l}0.265 \\
0.606\end{array}$} & 70 & 1 & \multirow{2}{*}{$\begin{array}{l}0.793 \\
0.373\end{array}$} \\
\hline $\begin{array}{l}\mathrm{H} / 0 \text { of use of } \\
\text { smokeless tobacco }\end{array}$ & 04 & 03 & $\begin{array}{l}0.503 \\
0.110-2.289\end{array}$ & & 05 & $\begin{array}{l}2.303 \\
0.599-8.855\end{array}$ & \\
\hline $\begin{array}{l}\text { No } \mathrm{H} / \mathrm{O} \text { of alcohol } \\
\text { intake }\end{array}$ & 103 & 134 & 1 & \multirow{2}{*}{$\begin{array}{l}3 \\
0.083\end{array}$} & 45 & 1 & \multirow{2}{*}{$\begin{array}{l}7.11 \\
0.007\end{array}$} \\
\hline $\mathrm{H} / \mathrm{O}$ alcohol intake & 30 & 61 & $\begin{array}{l}1.562 \\
0.941-2.594\end{array}$ & & 30 & $\begin{array}{l}2.288 \\
1.236-4.235\end{array}$ & \\
\hline $\mathrm{H} / \mathrm{O}$ regular exercise & 73 & 76 & 1 & \multirow[b]{2}{*}{$\begin{array}{l}8.08 \\
0.004\end{array}$} & 32 & 1 & \multirow[b]{2}{*}{$\begin{array}{l}2.87 \\
0.090\end{array}$} \\
\hline $\begin{array}{l}\text { No } \mathrm{H} / \mathrm{O} \text { regular } \\
\text { exercise }\end{array}$ & 60 & 119 & $\begin{array}{l}1.905 \\
1.218-2.977\end{array}$ & & 43 & $\begin{array}{l}1.634 \\
0.923-2.893\end{array}$ & \\
\hline BMI $<18.5 \mathrm{~kg} / \mathrm{m}^{2}$ & 31 & 13 & $\begin{array}{l}0.336 \\
0.160-0.707\end{array}$ & \multirow{2}{*}{$\begin{array}{l}8.64 \\
0.003\end{array}$} & 1 & $\begin{array}{l}0.077 \\
0.01-0.605\end{array}$ & \multirow{2}{*}{$\begin{array}{l}7.64 \\
0.005\end{array}$} \\
\hline BMI $18.5-22.9 \mathrm{~kg} / \mathrm{m}^{2}$ & 53 & 66 & 1 & & 22 & & \\
\hline BMI $23-24.9 \mathrm{~kg} / \mathrm{m}^{2}$ & 20 & 44 & $\begin{array}{l}1.766 \\
0.931-3.351\end{array}$ & $\begin{array}{l}3.06 \\
0.080\end{array}$ & 13 & $\begin{array}{l}1.565 \\
0.664-3.689\end{array}$ & $\begin{array}{l}1.06 \\
0.303\end{array}$ \\
\hline $\mathrm{BMI}>25 \mathrm{~kg} / \mathrm{m}^{2}$ & 29 & 72 & $\begin{array}{l}1.993 \\
1.135-3.499\end{array}$ & $\begin{array}{l}5.585 \\
0.015 \\
\end{array}$ & 39 & $\begin{array}{l}3.239 \\
1.622-6.468\end{array}$ & $\begin{array}{l}11.45 \\
0.000\end{array}$ \\
\hline Waist $<90 \mathrm{~cm}$ & 26 & 54 & 1 & \multirow{2}{*}{$\begin{array}{l}2.84 \\
0.091\end{array}$} & 26 & 1 & \multirow{2}{*}{$\begin{array}{l}5.85 \\
0.015\end{array}$} \\
\hline Waist $>90 \mathrm{~cm}$ & 107 & 141 & $\begin{array}{l}0.634 \\
0.373-1.079\end{array}$ & & 49 & $\begin{array}{l}0.457 \\
0.241-0.868\end{array}$ & \\
\hline $\begin{array}{l}\text { Blood Sugar }<140 \\
\mathrm{mg} / \mathrm{dl}\end{array}$ & 125 & 189 & 1 & $\begin{array}{l}1.67 \\
0.196\end{array}$ & 72 & 1 & $\begin{array}{l}0.091 \\
0.763\end{array}$ \\
\hline
\end{tabular}

Table 3 depicts the prevalence of CVD risk factors among normal, pre hypertensive and hypertensive of engineering and medical students. Among engineering students higher prevalence of CVD risk factors were seen in pre hypertensive compared to normotensive in above the age of 20 years, fruits and vegetable intake of less than 3 days a week, use of smokeless tobacco, H/O alcohol intake, lack of regular exercise and BMI of more than 25. But it was statistically significant for lack of exercise $(p<0.001)$ and BMI of more than $25(p=0.05)$. In case of medical students, higher prevalence of CVD risk factors were seen among pre hypertensive compared to normotensive above the age 20 years, consuming mixed diet, fruits intake of less than 3 days a week, $\mathrm{H} / \mathrm{O}$ alcohol intake, lack of regular exercise and BMI of more than 25 and waist circumference more than $90 \mathrm{~cm}$. But it was statistically significant for alcohol intake $(p=0.004)$, BMI of more than $25(\mathrm{p}=0.011)$ and waist circumference $(p<0.001)$. In both the medical and engineering students higher prevalence of CVD risk factors were seen compared to normotensive for all risk factors except for 
consumption of smokeless tobacco and blood sugar level of more than $140 \mathrm{mg} / \mathrm{dl}$.

Table 4 shows the association between risk factors of CVD and pre hypertensive and hypertensive. Higher risk of being pre hypertensive and hypertensive were noted above the age of 20 years $(\mathrm{OR}=4.32$ and 1.79$)$, fruit intake less than 3 days a week $(\mathrm{OR}=1.03$ and 1.33), smokers $(\mathrm{OR}=1.13$ and 1.68$)$, alcohol intake $(\mathrm{OR}=1.56$ and 2.28), lack of physical exercise $(\mathrm{OR}=1.90$ and 1.63), $\mathrm{BMI}$ of more than $25(\mathrm{OR}=1.99$ and 3.23).

But statistically significant difference was noted between pre hypertensive and normotensive for age $(\mathrm{p}<0.0001)$, lack of physical exercise $(\mathrm{p}=0.004)$ and BMI $(\mathrm{p}=0.015)$. Statistically significant difference was noted between normotensive and hypertensive for alcohol intake $(\mathrm{p}=0.007)$ and BMI $(\mathrm{p}<0.001)$.

\section{DISCUSSION}

This cross sectional study among 403 male undergraduate students showed about half of the students had pre hypertension and one fifth of the students had hypertension. Similar findings were observed by Hana T. Al-Majed et al among male college students in Kuwait, ${ }^{16}$ Balami Ad et al among Public University Students in Malaysia, ${ }^{11}$ and Shobha S Shetty et al among medical students in coastal Karnataka ${ }^{12}$ and Azza Mohamed Sarry El-Din et al. ${ }^{13}$ In contrast Pengpid $\mathrm{S}$ et al had reported among students of the University of the PhilippinesVisayas prevalence of pre hypertensive and hypertensive were $36.1 \%$ and $6.6 \%$ respectively among male students. ${ }^{17}$ Meta-analysis of Twenty-six articles, 20 crosssectional and 6 longitudinal studies by Xiaofan Guo et al showed the pooled prevalence of pre hypertension among males was 40 percent. ${ }^{18}$ The prevalence of cardiovascular risk factors was higher in pre hypertensive males for above the age of 20 years, vegetable intake less than 3 days a week, smokers, alcoholics, those who did not do regular exercise and postprandial blood sugar more than $140 \mathrm{mg} / \mathrm{dl}$. Similar findings was reported by Itamar Grotto et al reported the prevalence of cardiovascular risk factors was significantly higher in the prehypertension group than in the normotensive group for glucose level of more than $125 \mathrm{mg} / \mathrm{dl}$, BMI of $>25 \mathrm{~kg} / \mathrm{m}^{2}$, smokers and sedentary life style19. It was observed by other authors Greenlund KJ et al, ${ }^{6}$ and Vasan RS et al that persons with pre-hypertension had a higher prevalence of risk factors for CVD morbidity than those with a normal BP; including dyslipidemia, overweight and obesity, and diabetes mellitus. ${ }^{2}$

\section{Age and pre-hypertension}

The prevalence of cardiovascular risk factors was higher in pre-hypertensive males for above the age of 20 years. They were at higher risk of being pre hypertensive and the association between age and pre-hypertension was statistically significant. Similar findings of pre hypertension showing increasing trend with the increase in age was observed by $\mathrm{S}$ Srinivas et al among adult population of rural Andhra Pradesh. ${ }^{20}$ I.V. Fomin et al Age-specific prevalence of pre hypertension is increasing in the period from 10 to 60 years, preserving male priority. $^{21}$

\section{Course of study and pre hypertension}

In our study nearly half of the medical students had pre hypertension compared to engineering students which was slightly higher than engineering students but it was not statistically significant. Similar finding was reported by Monika Kuber Kotpalliwar et al among medical college students with $52 \%$ prevalence of pre hypertension $^{22}$ and Shobha S Shetty et al with $55.4 \%$ prevalence of pre hypertension among medical college students of coastal Karnataka. ${ }^{12}$ In contrast, P Das 1et al reported $21 \%$ prevalence of pre hypertension among under graduate students in West Bengal. ${ }^{23}$

\section{Diet and pre hypertension}

In our study no difference was noted in the prevalence of mixed diet (non-vegetarian) among pre hypertensive, hypertensive and normotensive. Positive association was seen for non-vegetarian diet and hypertension and not for pre hypertension and it was not statistically significant. Das, et $\mathrm{al}^{23}$ reported higher prevalence of HTN (13.5\%) among non-vegetarian students compared with those who reported consumption of vegetarian food $(8.7 \%)$.

\section{Fruit and vegetable intake}

In our study, higher prevalence of vegetable consumption less than 3 days a week was noted among pre hypertensive and not for less fruits consumption. Higher risk of being pre hypertensive was noted for both less fruit and vegetable consumption but it was not statistically significant. It has been stated that adherence to diet high in fruit and vegetables and low fat dairy products can reduce $\mathrm{BP}$ by $8-14 \mathrm{mmHg}^{24}$

\section{Smoking and pre hypertension}

In our study, among smokers $36.5 \%$ were normotensive, $18 \%$ were hypertensive and $27.3 \%$ were hypertensive. In contrast Hana T. Al-Majed et al reported among lower prevalence of pre-hypertensive (17.4\%), and hypertensive (17.9\%), among smokers. ${ }^{16}$ Das et $\mathrm{al}^{23}$ reported higher prevalence of hypertension among smokers $(58.6 \%)$ than nonsmokers $(5.3 \%)$. Our study odds ratio of 1.13 and 1.687 was noted pre hypertension and hypertension among smokers. The association was not statistically significant. Similar findings were observed with no significant association between smoking and blood pressure was not significant by Merlo $\mathrm{J}$ et al, ${ }^{25}$ John Meyer $\mathrm{C}$ et $\mathrm{al}^{26}$ and KarmiZarchi A A et al. ${ }^{27}$ Xiaofan Guo et al showed the pooled odds ratio was 1.13 for smoking among pre hypertensive. ${ }^{18}$ Balami A.D et al 
reported among Public University Students in Malaysia Ever smoker was significantly associated with prehypertension in bivariate but not multivariate analysis 28 . Rajesh Venkataraman et al had reported among rural population of South India with increase in smoking there was increase in the incidence of pre-hypertension and hypertension. ${ }^{29}$ Tsai PS et al also reported current smoking as significant risk factor for pre HTN and HTN among men. ${ }^{30}$

\section{Alcohol consumption and pre hypertension}

In our study, among students with H/o alcohol intake was higher both in pre hypertensive and hypertensive compared to normotensive. Even though alcoholics were at higher risk of being pre hypertensive and hypertensive statistically significant association was noted between alcoholics and hypertension and not for pre hypertension. Das, et $\mathrm{al}^{23}$ reported HTN was more prevalent $(14.28 \%)$ among students who consume alcohol than nonconsumers (12.8\%). Rajesh Venkataraman et al had reported among rural population of South India incidence of hypertension and pre-hypertension increases with the increase in the alcohol consumption. ${ }^{29}$ In our study odds ratio was 1,562 and 2.88 for alcohol intake among pre hypertensive and hypertensive. Statistically significant association was found between alcohol intake and hypertension and not for pre hypertension. Xiaofan Guo et al showed the pooled odds ratio was 0.98 for drinking among pre hypertensive. ${ }^{18}$

\section{Regular physical exercise and pre hypertension}

In our study, among students with H/o lack of physical exercise was higher both in pre hypertensive and hypertensive compared to normotensive. Even though, students with lack of physical exercise were at higher risk of being pre hypertensive and hypertensive, statistically significant association was noted between lack of physical exercise and pre -hypertension and not for hypertension. Das et al reported hypertensive prevalence was less $(12.5 \%)$ among persons who had done physical exercise and higher among those $(13.3 \%)$ who was not having any healthy habit like walking, jogging, etc. ${ }^{23}$ Balami A.D et al reported among Public University Students in Malaysia Low level of physical activity (54.3\%) was high among the students. Level of physical activity was not significantly associated with prehypertension in both bivariate and multivariate analysis. ${ }^{28}$ Felix $\mathrm{F}$ et al reported association between low physical activity and pre-hypertension with $\mathrm{OR}=2.6$ but not for hypertension. ${ }^{31}$ Similar findings were reported by Ejike $\mathrm{CE}$ et al the prevalence of hypertension increases when physical activity decreases. ${ }^{32}$

\section{Overweight and pre hypertension}

In our study, the prevalence of overweight was higher among pre-hypertensive (36\%) and hypertensive (51.6\%) compared to normotensives $(23.1 \%)$. Hana T. Al-Majed et al reported among college students in Kuwait with BMI $>25 \mathrm{~kg} / \mathrm{m}^{2} \quad 42.3 \%$ were normotensive, $62.1 \%$ were pre hypertensive and $75 \%$ were hypertensive 16 . In our study students who were overweight were at higher risk if being pre hypertensive compared to students with BMI of less than $25 \mathrm{~kg} / \mathrm{m}^{2}$ and it was found statistically significant. Xiaofan Guo et al had reported that individuals with pre-hypertension had higher body mass indices and waist circumferences than did people with normotension; waist circumferences showed greater relative differences. ${ }^{18}$ Das et al reported among students the Prevalence of HTN was found high among obese $(40.7 \%)$ and overweight $(15.9 \%)$ population than others. In our study odds ratio was 1.993 and 3.239 for overweight among pre hypertensive and hypertensive. ${ }^{23}$ Sougat Ray et $\mathrm{al}^{33}$ in his study among military adults reported pre-hypertension had significant positive association with BMI $>23 \mathrm{~kg} / \mathrm{m}^{2}$ with an OR of 1.75 . Positive association of overweight and obesity and pre hypertension was also supported by Greenlund KJ et al. ${ }^{6}$ Shobha S Shetty et al reported among medical students in coastal Karnataka significant correlation between prehypertension \& a raised BMI for male students. ${ }^{12}$ Balami A.D et al reported among Public University Students in Malaysia BMI status, over weight and obese persons were almost 6 times and 11 times respectively, more likely to have pre-hypertension than underweight. Body mass index status was significantly associated with prehypertension. ${ }^{28}$ Tesfaye $\mathrm{F}$ et al reported blood pressure was found to be associated with BMI, and BMI independently predicted blood pressure level in all study participants. ${ }^{34} \mathrm{~S}$. Asmathulla, reported BMI was found as an independent contributor for the development of PHT in their study population. ${ }^{35}$

\section{Waist circumference and pre hypertension}

In the present study even though the prevalence of waist circumference above $90 \mathrm{~cm}$ was higher for both pre hypertensive and hypertensive compared to normotensive, negative association was noted between increased waist circumference and pre hypertension and hypertension but it was not statistically significant.

\section{Blood sugar level and pre-hypertension}

In our study, among students with postprandial blood sugar $>140$ normotensive were $0.8 \%$, pre hypertensive were $3 \%$ and hypertensive were $3.9 \%$ similar findings was reported among students of Kuwait by Hana T. AlMajed et al where normotensive, pre-hypertensive and hypertensive was $0.9 \%, 1.9$ and $3.6 \%$ respectively. ${ }^{16}$ In the present study positive association was noted between blood sugar level and pre hypertension and hypertension and it was not statistically significant.

\section{CONCLUSION}

In conclusion, nearly half of the students were pre hypertensive. Pre-hypertension is a precursor of clinical 
hypertension and consequently of the cardiovascular disease (CVD). Higher prevalence of smoking, alcohol intake, lack of physical exercise, overweight and increased waist circumference and postprandial blood sugar more than $140 \mathrm{mg} / \mathrm{dl}$ was noted among pre hypertensive compared to normotensive. Higher risk of being pre hypertensive was noted among students above the age of 20 years; who consume less fruit and vegetable, smokers, alcoholics, overweight and lack of physical exercise. But statistically significant difference was noted for age, lack of physical exercise and overweight. Thus, our ultimate objective is to management of pre hypertension is to prevent hypertension and to reduce the CVD risk associated with pre hypertension.

Lack of regular physical activity, diet and overweight were reported by many studies as modifiable risk factors for pre hypertension. Education on dietary approach and weight reduction for prevention of pre hypertension and creating awareness among college students and introduction of physical activity in terms of sports or outdoor games as part of college teaching schedule will go a long way in the prevention of hypertension and cardiovascular risk factors in the adult population.

Funding: No funding sources

Conflict of interest: None declared

Ethical approval: The study was approved by the Institutional Ethics Committee

\section{REFERENCES}

1. Lewington S, Clarke R, Qizilbash N, Peto R, Collins R. Age-specific relevance of usual blood pressure to vascular mortality: a meta-analysis of individual data for one million adults in 61 prospective studies. Lancet. 2002;360:1903-13.

2. Vasan RS, Larson MG, Leip EP, Kannel WB, Levy D. Assessment of frequency of progression to hypertension in non-hypertensive participants in the framingham heart study: a cohort study. Lancet. 2001;358:1682-6.

3. Liszka HA, Mainous AG, King DE, Everett CJ, Egan BM. Pre-hypertension and cardiovascular morbidity. Ann Fam Med. 2005;3:294-9.

4. Svetkey LP. Management of Pre hypertension. Hypertension. 2005;45:1056-61.

5. Dieterle T, Sigle JP, Bengel G, Kiefer G, Brenneisen V, Martinab. Cardiovascular risk stratification in unselected primary care patients with newly detected arterial hypertension. Hypertens Res. 2010;33:607-15.

6. Greenlund KJ, Croft JB, Mensah GA. Prevalence of heart disease and stroke risk factors in persons with prhypertension in the United States,1999-2000. Arch Intern Med. 2004;164:2113-8.

7. Chobanian AV, Bakris GL, Black HR, Cushman WC, Green LA, Izzo JL Jr, et al. The seventh report of the Joint National Committee on Prevention,
Detection, Evaluation, and Treatment of High Blood Pressure: the JNC7 Report. JAMA. 2003;289(19):2560-72.

8. Vasan RS, Larson MG, Leip EP, Evans JC, O'Donnell CJ, Kannel WB et al. Impact of high normal blood pressure on the risk of cardiovascular disease. N Engl J Med. 2001;345:1291-7.

9. Chockalingam A, Ganesan N, Venkatesan S, Gnanavelu G, Subramaniam T, Jaganathan V et al. Patterns and predictors of pre-hypertension among "healthy" urban adults in India. Angiology. 2005;56:557-63.

10. Prabhakaran D, Shah P, Chaturvedi V, Ramakrishnan L, Manhapra A, Reddy KS. Cardiovascular risk factor prevalence among men in a large industry of northern India. Natl Med J India. 2005;18:59-65.

11. Balami AD, Salmiah MS, Nor Afiah MZ. Psychological determinants of pre-hypertension among first year undergraduate students in a public University in Malaysia. Malaysian J Pub Health Med. 2014;14(2):67-76.

12. Shetty SS, Nayak A. Prevalence of pre hypertension amongst medical students in coastal Karnataka. J Evolution Med Dent Sci. 2012;1(6):975-79.

13. El-Din AMS, Erfan M, Kandeel WA, Kamal S, ElShafy El Banna RA, Fouad WA. Prevalence of prehypertension and hypertension in a sample of Egyptian adults and its relation to obesity. Australian J Basic Appl Sci. 2012;6(13):481-9.

14. Physical Status: The use and interpretation of anthropometry. Report of a WHO expert committee. World Health Organ Tech Rep Ser. 1995;854:1-452.

15. Lean ME, Han TS, Morrison CE. Waist circumference as a measure for indicating need for weight management. BMJ. 1995;311(6998):158-61.

16. Al-Majed HT, Sadek AA. Pre-hypertension and hypertension in college students in Kuwait: A neglected issue. Family Community Med. 2012;19(2):105-12.

17. Pengpid S, Peltzer K, Ferrer AJ. Pre hypertension and associated factors among university students in the Philippines. Int $\mathrm{J}$ Adolesc Med Health. 2014;26(2):245-52.

18. Guo X, Zou L, Zhang X, Li J, Zheng L, Sun Z et al. A meta-analysis of the epidemiology, risk factors, and predictors of progression. Texas Heart Inst J. 2011;38(6):643-52.

19. Grotto I, Grossman E, Huerta M, Sharabi Y. Prevalence of pre hypertension and associated cardiovascular risk profiles among young israeli adults. Hypertension. 2006;48:254-59.

20. Srinivas S, Satyavaraprasad K, Ramdas, Krishna CPRS, Tajuddin, Prabhakar RR. Prevalence of prehypertension in adult population of rural Andhra Pradesh. Asian J Biomed Pharm Sci. 2013;3(23):458.

21. Fomin IV, Badin YV, Polyakov DS, Belenkov YN, Mareev VY, Ageev FT, Artemieva EG. Pre hypertension: how often this cardiovascular 
condition occurs in citizens of European Russia (EPOCH-AH, 2002-2007). Clin Med. 2013;5(2):3943.

22. Kotpalliwar MK, Wanjari A, Acharya S. Prevalence of pre hypertension in young healthy individuals and its associated risk factors. Indian J Med Health. 2013;2(3):242-8.

23. Das P, Basu M, Chowdhury K, Mallik S, Dhar G, Biswas A. Observational assessment and correlates to blood pressure of future physicians of Bengal. Niger J Clin Pract. 2013;16:433-8.

24. Collier SR, Landram MJ. Treatment of prehypertension: lifestyle and/or medication. Vascular Health Risk Management. 2012;8:613-9.

25. Merlo J, Asplund K, Lynch J, Rastan L, Dobson A. Population effects on individual systolic blood pressure: multivariate analysis of the WHO MONICA Project. Am J Epidemiol. 2004;159:116879.

26. John MC, Hanke M, Valzke H, Schumann A. Smoking status, obesity and hypertension in the general population sample: a cross sectional study. QJM. 2006;99(6):407-15.

27. Karimi ZAA, Gahangiri M. Prehypertension and hypertension in Iranian personnel. World Appl Sci J. 2010;10(8):892-8.

28. Balami AD, Salmiah MS, Nor Afiah MZ. Association of behavioural factors, body mass index status, family history of hypertension and prehypertension among. A public university student in Malaysia. Intern $\mathbf{J}$ Pub Heal Clin Sci. 2014;1(2):109-20.

29. Venkataraman R, Satish Kumar BP, Kumaraswamy M, Singh R, Pandey M, Tripathi P et al. Smoking, alcohol and hypertension. Int $\mathrm{J}$ Pharm Pharm Sci. 2013;5(4):28-32.
30. Tsai PS, Ke TL, Huang CJ, Jen-Chena T, PingLinga C, Shu-Yia W et al. Prevalence and determinants of prehypertension status in the Taiwanese general population. J Hypertens. 2005;23:1355-6.

31. Widjaja FF, Santoso LA, Barus NRV, Pradana GA, Estetika C. Prehypertension and hypertension among young Indonesian adults at a primary health care in a rural area. Med J Indones. 2013;22(1):3945.

32. Ejike CE, Ugwu CE, Ezeanyika LU. Variations in the prevalence of point (pre) hypertension in a Nigerian school-going adolescent population living in a semi-urban and an urban area. BMC Pediatrics. 2010;10:13-7.

33. Ray S, Kulkarni B, Sreenivas A. Prevalence of pre hypertension in young military adults \& its association with overweight \& dyslipidaemia. Indian J Med Res. 2011;134(2):162-7.

34. Tesfaye F, Nawi NG, Van Minh H, Byass P, Berhane Y, Bonita R, Wall S. Association between body mass index and blood pressure across three populations in Africa and Asia. J Hum Hypertens. 2007;21:28-37.

35. Asmathulla S, Rajagovindan D, Sathyapriya V, Pai B. Prevalence of prehypertension and its relationship to cardiovascular disease risk factors in Puducherry. Indian J Physiol Pharmacol. 2011;55(4):343-50.

Cite this article as: Logaraj M, Madhavan RSD, Balaji R. Prevalence of pre hypertension and its association to risk factors for cardiovascular diseases among male undergraduate students in Chennai. Int $\mathbf{J}$ Community Med Public Health 2016;3:542-50. 